\title{
Performance Analysis of Macrodiversity MIMO Systems with MMSE and ZF Receivers in Flat Rayleigh Fading
}

\author{
Dushyantha A. Basnayaka, Student Member, IEEE, Peter J. Smith, Senior Member, IEEE and Phillipa A. \\ Martin, Senior Member, IEEE
}

\begin{abstract}
Consider a multiuser system where an arbitrary number of users communicate with a distributed receive array over independent Rayleigh fading paths. The receive array performs minimum mean squared error (MMSE) or zero forcing (ZF) combining and perfect channel state information is assumed at the receiver. This scenario is well-known and exact analysis is possible when the receive antennas are located in a single array. However, when the antennas are distributed, the individual links all have different average signal to noise ratio (SNRs) and this is a much more challenging problem. In this paper, we provide approximate distributions for the output SNR of a $\mathrm{ZF}$ receiver and the output signal to interference plus noise ratio (SINR) of an MMSE receiver. In addition, simple high SNR approximations are provided for the symbol error rate (SER) of both receivers assuming $M$-PSK or $M$-QAM modulations. These high SNR results provide array gain and diversity gain information as well as a remarkably simple functional link between performance and the link powers.
\end{abstract}

Index Terms-Macrodiversity, MMSE, ZF, Outage probability, Optimum combining, Zero-Forcing, Network MIMO, CoMP.

\section{INTRODUCTION}

With the advent of space diversity systems, decoupling users through channel aware signal processing techniques in the presence of multiple access interference (MAI) and noise has become an integral part of the system design. There are various processing techniques now widely adopted in research and standards [1]. Among them, linear combining methods are popular for their simplicity despite the fact that they are not optimum in a maximum likelihood sense. Two key linear combiners are zero forcing (ZF) and minimum-mean squarederror (MMSE). Although they are not optimal, the MMSE receiver satisfies an alternative criterion, i.e., it minimizes the mean squared error (MSE) and ZF is known to eliminate MAI completely.

The performance analysis of such linear receivers is of great interest in wireless communication [2] as it provides a baseline link level performance metric for the system. Today, performance results for MMSE/ZF receivers are well known for microdiversity systems where co-located diversity antennas at the base station communicate with distributed users [3], [4], [7]. Macro-scale diversity combining has recently become

D. A. Basnayaka, P. J. Smith and P. A. Martin are with the Department of Electrical and Computer Engineering, University of Canterbury, Christchurch, New Zealand. E-mail:\{dush, p.smith, p.martin\}@elec.canterbury.ac.nz.

D. A. Basnayaka is supported by a University of Canterbury International Doctoral Scholarship. more common from a variety of perspectives [8], [9]. Any system where both transmit and receive antennas are widely separated can be interpreted as a macrodiversity multiple input multiple output (MIMO) system. They occur naturally in network MIMO systems [9], [10] and collaborative MIMO concepts [11, p. 69] and [12].

The performance of macrodiversity systems has been investigated via simulation [13], but very few analytical results appear to be available. The reason for the lack of results is the complexity of the channel matrix that arises in macrodiversity systems. When the receive antennas are co-located, classical models and Kronecker correlation matrix has a Wishart form. Here, extensive results in multivariate statistics can be leveraged and performance analysis is now well advanced. In contrast, the macrodiversity case violates the Wishart assumptions and there is no such distribution in the literature for macrodiversity channel matrices for finite size systems. This makes most of the analytical work extremely difficult. The analytical complexity is clearly evident even in the simplest case of a dual source scenario [14].

Despite this complexity, some analytical results are available for the dual user case in [14] for macrodiversity MMSE and ZF receivers. In [14], they consider the statistical properties of the output signal to interference plus noise ratio (SINR)/signal to noise ratio (SNR) of MMSE and ZF receivers respectively and obtain high SNR approximations of the symbol error rate (SER). In [15], the SER performance of macrodiversity maximal ratio combining (MRC) has been exactly derived for arbitrary numbers of users and antenna configurations. The ergodic sum capacity of the macrodiversity MIMO multiple access channel is considered in [16] where tight approximations of ergodic sum capacity are derived in a compact form. Rayleigh fading is assumed for finite system sizes in [14], [15] and [16]. One of the analytical techniques used in [16] is also used here. In [16], sum capacity in logarithmic form is expressed as an exponential and ergodic sum capacity is then written as the mean of a ratio of quadratic forms. In this work, we have a very different starting point and consider the characteristic function (CF) of the SNR/SINR. The exponential in the $\mathrm{CF}$ also leads to a mean of a ratio of quadratic forms. Hence, the two studies produce similar ratios at this point in the analysis and the same technique, namely a Laplace type approximation [27], is employed both here and in [16] to simplify the result. Note that the analysis leading up to the ratio of quadratic forms and following 
the Laplace approximation is quite specific to the individual problems considered. As a result, [16] gives approximate results for ergodic capacity and here, approximate SER results and SNR/SINR distributions are obtained. Note that some of the quadratic forms encountered here are of a different form to those in [16].

On an another front, an asymptotic large random matrix approach is employed to derive a deterministic equivalent to the ergodic sum capacity in [17]. Similarly, an asymptotic approach is used to study cellular systems with multiple correlated base station (BS) and user antennas in [18], [19].

In this paper, we extend the results in [14] to more general user and antenna configurations. In particular, the contributions made are as follows:

1. We derive the approximate probability distribution function (PDF) and cumulative distribution function (CDF) of the output SINR/SNR of MMSE/ZF receivers. The approximate cumulative distribution functions are shown to have a remarkably simple form as a generalized mixture of exponentials.

2. High SNR approximations for the SER of MMSE/ZF receivers are derived for a range of modulations and these results are used to derive diversity order and array gain results. The high SNR results are simple, have a compact form and can be used to gain further insights into the effects of channel distribution information (CDI) on the performance of macrodiversity MIMO systems.

The rest of the paper is laid out as follows. Motivational example for this work is given in Sec. II. Sec. III describes the system model and receiver types. Sec. IV provides preliminary results which will be used throughout the paper. The main analysis is given in Secs. V and VI. Secs. VII and VIII give numerical results and conclusions, respectively.

\section{Motivational ExAmple}

Consider a hypothetical MIMO spatial multiplexing communication system with three transmit and three receive antennas. The complex channel gain, $h_{i k}$, represents the fading coefficient between transmit antenna $k$ and receive antenna $i$. The average link gain between the same antennas is given by $P_{i k}$. In the following, we consider two cases of this MIMO system.

1) Macrodiversity System: In this system, we assume all $P_{i k} \mathrm{~s}$ are different. This case arises in MIMO systems where both transmit antennas and receive antennas are widely separated. Then, we consider a particular realization of the power matrix $\boldsymbol{P}=\left(P_{i k}\right)$ for $i, k=1, \ldots, 3$, given as

$$
\boldsymbol{P}_{M}=\left(\begin{array}{lll}
0.3500 & 0.0117 & 0.1225 \\
0.6292 & 0.9282 & 0.0741 \\
0.0208 & 0.0601 & 0.8035
\end{array}\right) .
$$

2) Point-to-Point System: We assume that all the $P_{i k}$ are the same in this system. This case arises in single user MIMO links with closely spaced transmit and receive antennas. The point-to-point power matrix is given as

$$
\boldsymbol{P}_{P}=\left(\begin{array}{lll}
0.3333 & 0.3333 & 0.3333 \\
0.3333 & 0.3333 & 0.3333 \\
0.3333 & 0.3333 & 0.3333
\end{array}\right) .
$$

Note that the actual values of $\boldsymbol{P}_{P}$ and $\boldsymbol{P}_{M}$ are unimportant, it is the comparison between equal and unequal powers that is relevant. Furthermore, we assume $\mathrm{ZF}$ receive combining is used to spatially separate each independent stream. Each column of the channel power matrix is normalized so the sum of its power elements is equal to unity and equal power loading is assumed for each data stream. This ensures that the average receive power of each stream is constant and the performance of data streams can be compared. If we consider the uncoded SER of each stream in both cases, it is clear that the SER of all three streams is the same in the point-to-point case due to the symmetry of the channel power matrix, P. However, in the macrodiversity case, the picture is not so clear as shown in Fig. 11 Due to the structure of the power matrix, $\boldsymbol{P}_{M}$, the curves exhibit substantial performance gaps in terms of array gain. This effect can be called the macrodiversity effect and is a function of the slow fading information or the statistical information of the channel matrix

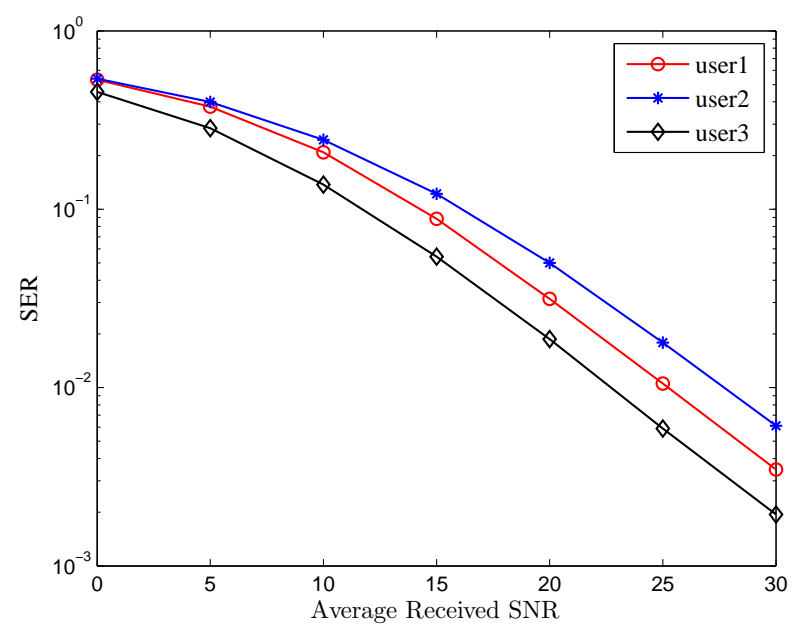

Fig. 1. Simulated SER results for the macrodiversity case with QPSK modulation and a $\mathrm{ZF}$ receiver. Results are given for all 3 users.

In this paper, we develop analytical tools to understand the macrodiversity effect on SER. We also construct some accompanying statistical measures which may be widely useful for analyzing macrodiversity systems in the context of the multiuser MIMO multiple access channel.

\section{System ModeL}

In this section, we present the generic system model which is considered throughout this paper. The multiuser MIMO system investigated in this paper consists of $N$ distributed single antenna users communicating with $n_{R}$ distributed receive antennas in an independent flat Rayleigh fading environment. The $\mathcal{C}^{n_{R} \times 1}$ receive vector is given by

$$
r=H s+n,
$$


where the $\mathcal{C}^{N \times 1}$ data vector, $\boldsymbol{s}=\left(s_{1}, s_{2}, \ldots, s_{N}\right)^{T}$, contains the transmitted symbols from the $N$ users and it is normalized, so that $E\left\{\left|s_{i}\right|^{2}\right\}=1$ for $i=1,2, \ldots, N$. $\boldsymbol{n}$ is the $\mathcal{C}^{n_{R} \times 1}$ additive-white-Gaussian-noise (AWGN) vector, $\boldsymbol{n} \sim \mathcal{C N}\left(\mathbf{0}, \sigma^{2} \boldsymbol{I}\right)$, which has independent entries with $E\left\{\left|n_{i}\right|^{2}\right\}=\sigma^{2}$, for $i=1,2, \ldots, n_{R}$. The channel matrix contains independent elements, $H_{i k} \sim \mathcal{C N}\left(0, P_{i k}\right)$, where $E\left\{\left|H_{i k}\right|^{2}\right\}=P_{i k}$. A typical macrodiversity MU-MIMO multiple access channel (MAC) is shown in Fig. 2, where it is clear that the geographical spread of users and antennas creates a channel matrix $\boldsymbol{H}$, which has independent entries with different $P_{i k}$ values. We define the $\mathcal{C}^{n_{R} \times N}$ matrix, $\boldsymbol{P}=\left\{P_{i k}\right\}$, which holds the average link powers due to shadowing, path fading, etc.

By assuming that perfect channel state information is available

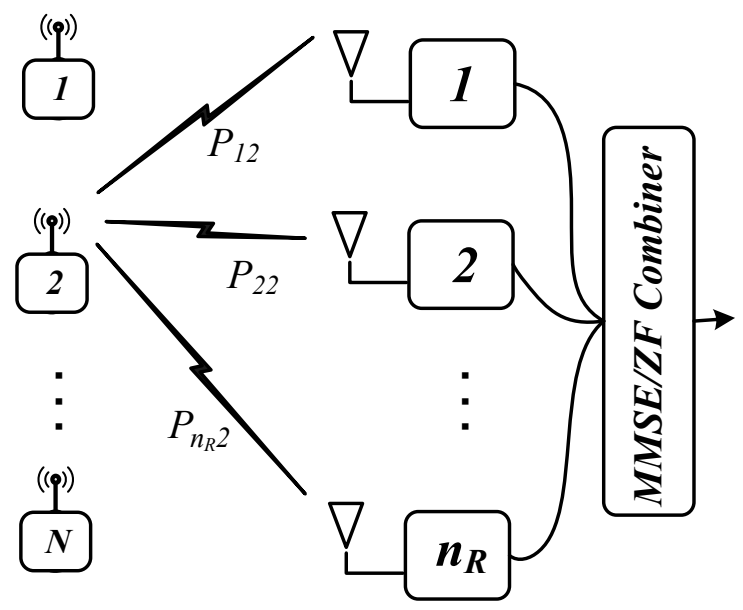

Fig. 2. System diagram. To reduce clutter, only paths from a single source are shown.

at the receiver side, we consider a system where channel adaptive linear combining is performed at the receiver to suppress multiple access interference [1]. Therefore, the $\mathcal{C}^{N \times 1}$ combiner output vector is $\tilde{\boldsymbol{r}}=\boldsymbol{V}^{H} \boldsymbol{r}$, where $\boldsymbol{V}$ is an $\mathcal{C}^{n_{R} \times N}$ weight matrix. In this work, we consider two well-known linear combining schemes: MMSE and ZF. The structure of $V$ and the resulting output SINR/SNR for MMSE/ZF schemes are well-known and are given below. Without loss of generality, we assume that the index of the desired user is $i=1$. The combining vector and output SINR of the MMSE receiver for user 1 are given by [3], [20] as

$$
\begin{gathered}
\boldsymbol{v}_{1}=\left(\boldsymbol{H} \boldsymbol{H}^{H}+\sigma^{2} \boldsymbol{I}\right)^{-1} \boldsymbol{h}_{1}, \\
\text { SINR }=\boldsymbol{h}_{1}^{H} \boldsymbol{R}^{-1} \boldsymbol{h}_{1},
\end{gathered}
$$

where

$$
\boldsymbol{R}=\sum_{k \neq i}^{N} \boldsymbol{h}_{k} \boldsymbol{h}_{k}^{H}+\sigma^{2} \boldsymbol{I},
$$

and $\boldsymbol{H}=\left(\boldsymbol{h}_{1}, \boldsymbol{h}_{2}, \ldots, \boldsymbol{h}_{N}\right)$. Defining $\boldsymbol{v}_{2}, \ldots, \boldsymbol{v}_{N}$ similarly gives $\boldsymbol{V}=\left(\boldsymbol{v}_{1}, \boldsymbol{v}_{2}, \ldots, \boldsymbol{v}_{N}\right)$. The vectors, $\boldsymbol{h}_{k}$, clearly play an important role in MMSE combining and it is useful to define the covariance matrix of $\boldsymbol{h}_{k}$ by $\boldsymbol{P}_{k}=E\left\{\boldsymbol{h}_{k} \boldsymbol{h}_{k}^{H}\right\}=$ $\operatorname{diag}\left(P_{1 k}, P_{2 k}, \ldots, P_{n_{R} k}\right)$. From [4], [7], the combining matrix, $\boldsymbol{V}$, and output SNR of the ZF receiver for $n_{R} \geq N$ are given by

$$
\boldsymbol{V}=\boldsymbol{H}\left(\boldsymbol{H}^{H} \boldsymbol{H}\right)^{-1}
$$

and

$$
\mathrm{SNR}=\frac{1}{\sigma^{2}\left[\left(\boldsymbol{H}^{H} \boldsymbol{H}\right)^{-1}\right]_{11}}
$$

where $[\boldsymbol{B}]_{11}$ indicates the $(1,1)^{t h}$ element of matrix $\boldsymbol{B}$.

\section{Preliminaries}

In this section, we state some useful results which will be used extensively throughout the paper.

Let $\boldsymbol{A}=\left(a_{i k}\right)$ be an $m \times n$ rectangular matrix over the commutative ring, $m \leq n$. The permanent of $\boldsymbol{A}$, written $\operatorname{Perm}(\boldsymbol{A})$, is defined by

$$
\operatorname{Perm}(\boldsymbol{A})=\sum_{\sigma} a_{1, \sigma_{1}} a_{2, \sigma_{2}} \ldots a_{m, \sigma_{m}},
$$

where the summation extends over all one-to-one functions from $\{1, \ldots, m\}$ to $\{1, \ldots, n\}$. The sequence $\left(a_{1, \sigma_{1}} a_{2, \sigma_{2}} \ldots a_{m, \sigma_{m}}\right)$ is called a diagonal of $\boldsymbol{A}$, and the product $a_{1, \sigma_{1}} a_{2, \sigma_{2}} \ldots a_{m, \sigma_{m}}$ is a diagonal product of $\boldsymbol{A}$. Thus, the permanent of $\boldsymbol{A}$ is the sum of all diagonal products of $A$.

Lemma 1. 16 Let $\boldsymbol{X}$ be an $m \times n$ random matrix with,

$$
\boldsymbol{A}=E\{\boldsymbol{X} \circ \boldsymbol{X}\} \triangleq\left(\begin{array}{ccc}
E\left\{\left|X_{11}\right|^{2}\right\} & \ldots & E\left\{\left|X_{1 n}\right|^{2}\right\} \\
E\left\{\left|X_{21}\right|^{2}\right\} & \ldots & E\left\{\left|X_{2 n}\right|^{2}\right\} \\
\ldots & \ldots & \ldots \\
E\left\{\left|X_{m 1}\right|^{2}\right\} & \ldots & E\left\{\left|X_{m n}\right|^{2}\right\}
\end{array}\right) \text {, }
$$

where o represents the Hadamard product. With this notation, the following identity holds.

$$
E\left\{\left|\boldsymbol{X}^{H} \boldsymbol{X}\right|\right\}= \begin{cases}\operatorname{perm}(\boldsymbol{A}) & m=n \\ \operatorname{Perm}(\boldsymbol{A}) & m>n,\end{cases}
$$

where perm(.) and Perm(.) are the permanent of a square matrix and rectangular matrix respectively as defined in [21].

Corollary 1. [16] Let $\boldsymbol{X}$ be an $m \times n$ random matrix with, $E\{\boldsymbol{X} \circ \boldsymbol{X}\}=\boldsymbol{A}$, where $\boldsymbol{A}$ is an $m \times n$ deterministic matrix and $m>n$. If the $m \times m$ deterministic matrix $\boldsymbol{\Sigma}$ is diagonal, then the following identity holds,

$$
E\left\{\left|\boldsymbol{X}^{H} \boldsymbol{\Sigma} \boldsymbol{X}\right|\right\}=\operatorname{Perm}(\boldsymbol{\Sigma} \boldsymbol{A}) \text {. }
$$

Next, we present three axiomatic identities for permanents [16].

- Axiom 1: For an empty matrix, $\boldsymbol{A}$,

$$
\operatorname{Perm}(\boldsymbol{A})=1 \text {. }
$$


- Axiom 2: Let $\boldsymbol{A}$ be an arbitrary $m \times n$ matrix, then

$$
\sum_{\sigma} \operatorname{Perm}\left((\boldsymbol{A})^{\sigma_{0, n}}\right)=\sum_{\sigma} \operatorname{Perm}\left((\boldsymbol{A})_{\sigma_{0, m}}\right)=1 .
$$

- Axiom 3: Let $\boldsymbol{A}$ be an arbitrary $m \times n$ matrix, then

$$
\sum_{\sigma} \operatorname{Perm}\left((\boldsymbol{A})_{\sigma_{k, m}}\right)=\sum_{\sigma} \operatorname{Perm}\left((\boldsymbol{A})^{\sigma_{k, n}}\right),
$$

where $\sigma_{k, n}$ is an ordered subset of $\{n\}=\{1, \ldots, n\}$ of length $k$ and the summation over all such subsets. $\boldsymbol{X}_{\sigma_{\ell, n}}$ denotes the principal submatrix of $\boldsymbol{X}$ formed by taking only the rows and columns indexed by $\sigma_{\ell, n}$.

In general, $\boldsymbol{X}_{\sigma_{\ell, n}}^{\mu_{\ell, n}}$ denotes the submatrix of $\boldsymbol{X}$ formed by taking only the rows and columns indexed by $\sigma_{\ell, n}$ and $\mu_{\ell, n}$ respectively, where $\sigma_{\ell, n}$ and $\mu_{\ell, n}$ are length $\ell$ subsets of $\{1,2, \ldots, n\}$. If either $\sigma_{\ell, n}$ or $\mu_{\ell, n}$ contain the complete set, the corresponding subscript/superscript may be dropped. When $\sigma_{\ell, n}=\mu_{\ell, n}$, only one subscript/superscript may be shown for brevity.

\section{ZF ANALYSIS}

In this section, we derive an approximate $\mathrm{CDF}$ for the output SNR of a ZF receiver, a high SNR approximation to SER and also consider some special cases. The following PDFs for the columns of the channel matrix are used throughout the analysis.

$$
f\left(\boldsymbol{h}_{k}\right)=\frac{1}{\pi^{n_{R}}\left|\boldsymbol{P}_{k}\right|} e^{-\boldsymbol{h}_{k}^{H} \boldsymbol{P}_{k}^{-1} \boldsymbol{h}_{k}},
$$

for $k=1,2, \ldots, N$.

\section{A. CDF Approximations}

The output SNR of a ZF receiver in (8) can be written as

$$
\begin{aligned}
\tilde{Z} & =\frac{1}{\sigma^{2}} \boldsymbol{h}_{1}^{H}\left(\boldsymbol{I}-\boldsymbol{H}_{2}\left(\boldsymbol{H}_{2}^{H} \boldsymbol{H}_{2}\right)^{-1} \boldsymbol{H}_{2}^{H}\right) \boldsymbol{h}_{1} \\
& =\frac{1}{\sigma^{2}} \boldsymbol{h}_{1}^{H} \boldsymbol{M} \boldsymbol{h}_{1},
\end{aligned}
$$

where $\boldsymbol{M}=\boldsymbol{I}-\boldsymbol{H}_{2}\left(\boldsymbol{H}_{2}^{H} \boldsymbol{H}_{2}\right)^{-1} \boldsymbol{H}_{2}^{H}$ and $\boldsymbol{H}_{2}$ is $\boldsymbol{H}$, with $h_{1}$ removed. Following the analysis in [14], the characteristic function $(\mathrm{CF})$ of $\tilde{Z}$ is given by

$$
\phi_{\tilde{Z}}(t)=E\left\{e^{j t \tilde{Z}}\right\}=E\left\{e^{\frac{j t}{\sigma^{2}} \boldsymbol{h}_{1}^{H} \boldsymbol{M} \boldsymbol{h}_{1}}\right\} .
$$

Conditioning on $\boldsymbol{H}_{2}$, the expectation over $\boldsymbol{h}_{1}$ in (18) can be solved as in [14] to obtain

$$
\phi_{\tilde{Z}}\left(t \mid \boldsymbol{H}_{2}\right)=\frac{1}{\left|\boldsymbol{I}-j t \frac{1}{\sigma^{2}} \boldsymbol{M} \boldsymbol{P}_{1}\right|} .
$$

Substituting for $M$ in (19) gives

$$
\begin{aligned}
\phi_{\tilde{Z}}\left(t \mid \boldsymbol{H}_{2}\right) & =\frac{1}{\left|\boldsymbol{I}-\frac{j t}{\sigma^{2}} \boldsymbol{P}_{1}+\frac{j t}{\sigma^{2}} \boldsymbol{H}_{2}\left(\boldsymbol{H}_{2}^{H} \boldsymbol{H}_{2}\right)^{-1} \boldsymbol{H}_{2}^{H} \boldsymbol{P}_{1}\right|} \\
& =\frac{1}{|\boldsymbol{D}|\left|\boldsymbol{I}+\frac{j t}{\sigma^{2}} \boldsymbol{H}_{2}\left(\boldsymbol{H}_{2}^{H} \boldsymbol{H}_{2}\right)^{-1} \boldsymbol{H}_{2}^{H} \boldsymbol{P}_{1} \boldsymbol{D}^{-1}\right|} \\
& =\frac{\left|\boldsymbol{H}_{2}^{H} \boldsymbol{H}_{2}\right|}{|\boldsymbol{D}|\left|\boldsymbol{H}_{2}^{H} \boldsymbol{H}_{2}+\frac{j t}{\sigma^{2}} \boldsymbol{H}_{2}^{H} \boldsymbol{P}_{1} \boldsymbol{D}^{-1} \boldsymbol{H}_{2}\right|},
\end{aligned}
$$

where $\boldsymbol{D}=\boldsymbol{I}-\frac{1}{\sigma^{2}} j t \boldsymbol{P}_{1}$. Simplifying (22) using the result $\boldsymbol{I}+\frac{j t}{\sigma^{2}} \boldsymbol{P}_{1} \boldsymbol{D}^{-1}=\boldsymbol{D}^{-1}$ gives

$$
\phi_{\tilde{Z}}\left(t \mid \boldsymbol{H}_{2}\right)=\frac{\left|\boldsymbol{H}_{2}^{H} \boldsymbol{H}_{2}\right|}{|\boldsymbol{D}|\left|\boldsymbol{H}_{2}^{H} \boldsymbol{D}^{-1} \boldsymbol{H}_{2}\right|} .
$$

The full CF can then be obtained by averaging the conditional $\mathrm{CF}$ in (23), to give

$$
\phi_{\tilde{Z}}(t)=\frac{1}{|\boldsymbol{D}|} E\left\{\frac{\left|\boldsymbol{H}_{2}^{H} \boldsymbol{H}_{2}\right|}{\left|\boldsymbol{H}_{2}^{H} \boldsymbol{D}^{-1} \boldsymbol{H}_{2}\right|}\right\},
$$

where expectation is over $\boldsymbol{H}_{2}$. An exact analysis of (24) is extremely cumbersome. However, for the dual source scenario where $N=2$, (24) can be solved in closed form [26]. Even for $N=2$, the resulting exact expressions are complex. Hence, for arbitrary $N$ we use a Laplace type approximation as in [14] to approximate and simplify the CF. This approximation has some motivation in the work of [27] and [16], [28]. It can also be thought of as a first order delta expansion [29]. Further insight into the use of the Laplace approximation can be gained from the case where $n_{R}$ is large and $\mathrm{N}=2$. Here, $\boldsymbol{H}_{2}$ is a column vector and the numerator and denominator in (25) are standard quadratic forms. Normalizing both numerator and denominator by dividing by $n_{R}$ leads to a ratio where both quadratic forms tend to constants as long as the conditions of a version of the weak law of large numbers hold. In this case, 25) becomes asymptotically exact. Hence, the stabilizing effect of averaging in the numerator and denominator is the motivation for the use of the Laplace approximation. This approach gives

$$
\phi_{\tilde{Z}}(t) \simeq \frac{1}{|\boldsymbol{D}|} \frac{E\left\{\left|\boldsymbol{H}_{2}^{H} \boldsymbol{H}_{2}\right|\right\}}{E\left\{\left|\boldsymbol{H}_{2}^{H} \boldsymbol{D}^{-1} \boldsymbol{H}_{2}\right|\right\}} .
$$

It is worth noting here that if the diagonal matrix, $\boldsymbol{P}_{1}$, is a scaled identity matrix, the approximation in (25) becomes exact regardless of the power matrix of $\boldsymbol{H}_{2}$. In contrast to microdiversity ZF, when $\boldsymbol{P}_{1}$ is not a scaled identity matrix, then $D$ in (24) is not a scaled identity and cannot be factorized out of the determinant. As a result, the CF in (24) depends on the expected value over $\boldsymbol{H}_{2}$ and so the statistical performance changes according to the power matrix of $\boldsymbol{H}_{2}$ (i.e., according to the macrodiversity power profile of the interference). 
Applying Lemma 1 and Corollary 1 to (25) gives

$$
\phi_{\tilde{Z}}(t) \simeq \frac{\operatorname{Perm}\left(\boldsymbol{Q}_{2}\right)}{|\boldsymbol{D}| \operatorname{Perm}\left(\boldsymbol{D}^{-1} \boldsymbol{Q}_{2}\right)},
$$

where the $n_{R} \times(N-1)$ matrix $\boldsymbol{Q}_{2}$ is defined by $\boldsymbol{Q}_{2}=$ $E\left\{\boldsymbol{H}_{2} \circ \boldsymbol{H}_{2}\right\} . \boldsymbol{P}=\left(\boldsymbol{p}_{1} \boldsymbol{Q}_{2}\right)$, and $\boldsymbol{p}_{1}=\left(P_{11}, P_{21}, \ldots, P_{n_{R} 1}\right)^{T}$. From Appendix A, the denominator in (26) can be expanded as

$$
|\boldsymbol{D}| \operatorname{Perm}\left(\boldsymbol{D}^{-1} \boldsymbol{Q}_{2}\right)=\sum_{i=0}^{L}(-j t)^{i} \tilde{\varphi}_{i}
$$

where

$$
\tilde{\varphi}_{i}=\sum_{\sigma} \operatorname{Tr}_{i}\left(\left(\boldsymbol{P}_{1}\right)_{\bar{\sigma}_{L, n_{R}}}\right) \operatorname{perm}\left(\left(\boldsymbol{Q}_{2}\right)_{\sigma_{N-1, n_{R}}}^{\{N-1\}}\right)\left(\sigma^{2}\right)^{-i},
$$

and $\operatorname{Tr}_{i}($.$) are elementary symmetric functions defined in [22,$ 1.2.12]. Since perm $\left(\left(\boldsymbol{Q}_{2}\right)_{\sigma_{N-1, n_{R}}}^{\{N-1\}}\right)$ is independent of $t$, it is clear from $(28)$ that $|\boldsymbol{D}| \operatorname{Perm}\left(\boldsymbol{D}^{-1} \boldsymbol{Q}_{2}\right)$ is a polynomial in $t$ of degree $L$. Hence, (26) becomes

$$
\begin{aligned}
\phi_{\tilde{Z}}(t) & \simeq \frac{\operatorname{Perm}\left(\boldsymbol{Q}_{2}\right)}{\sum_{i=0}^{L}(-j t)^{i} \tilde{\varphi}_{i}} \\
& =\frac{\operatorname{Perm}\left(\boldsymbol{Q}_{2}\right)}{\tilde{\varphi}_{L} \sum_{i=0}^{L}\left(\frac{\tilde{\varphi}_{i}}{\tilde{\varphi}_{L}}\right)(-j t)^{i}} \\
& =\frac{\operatorname{Perm}\left(\boldsymbol{Q}_{2}\right)}{\tilde{\varphi}_{L} \prod_{i=1}^{L}\left(\tilde{\omega}_{i}-j t\right)} \\
& =\frac{\operatorname{Perm}\left(\boldsymbol{Q}_{2}\right)}{\tilde{\varphi}_{L}} \sum_{i=1}^{L} \frac{\tilde{\eta}_{i}}{\tilde{\omega}_{i}-j t},
\end{aligned}
$$

where $\tilde{\omega}_{i}$ are the roots of the denominator polynomial in (30). These roots can be computed using standard root finding programs. Note that the roots are all positive, $\tilde{\omega}_{i}>0$ for all $i$, from Descarte's rule of signs and

$$
\tilde{\eta}_{i}=\frac{1}{\prod_{k \neq i}^{n_{R}}\left(\tilde{\omega}_{k}-\tilde{\omega}_{i}\right)} .
$$

It is clear from (26) that $\phi_{\tilde{Z}}(0)=1$, since $\boldsymbol{D}=\boldsymbol{I}$ when $t=0$. Therefore, the CF will produce a valid PDF after inversion [23]. From [24], the PDF and CDF of $\tilde{Z}$ are given by

$$
\begin{gathered}
f_{\tilde{Z}}(z)=\frac{1}{2 \pi} \int_{-\infty}^{\infty} \phi_{\tilde{Z}}(t) e^{-j t z} d t, \\
F_{\tilde{Z}}(z)=\frac{1}{2 \pi} \int_{0}^{z} \int_{-\infty}^{\infty} \phi_{\tilde{Z}}(t) e^{-j t x} d t d x .
\end{gathered}
$$

Substituting (32) in (34) gives the approximate PDF

$$
\hat{f}_{\tilde{Z}}(z)=\frac{\operatorname{Perm}\left(\boldsymbol{Q}_{2}\right)}{2 \pi \tilde{\varphi}_{L}} \sum_{i=1}^{L} \tilde{\eta}_{i} \int_{-\infty}^{\infty} \frac{e^{-j t z}}{\tilde{\omega}_{i}-j t} d t .
$$

Applying the integral identity from [25, eq. 7, 3.382], we obtain the approximate PDF of $\tilde{Z}$ as

$$
\hat{f}_{\tilde{Z}}(z)=\frac{\operatorname{Perm}\left(\boldsymbol{Q}_{2}\right)}{\tilde{\varphi}_{L}} \sum_{i=1}^{L} \tilde{\eta}_{i} e^{-\tilde{\omega}_{i} z},
$$

and the approximate $\mathrm{CDF}$ of $\tilde{Z}$ becomes

$$
\hat{F}_{\tilde{Z}}(z)=\frac{\operatorname{Perm}\left(\boldsymbol{Q}_{2}\right)}{\tilde{\varphi}_{L}} \sum_{i=1}^{L} \frac{\tilde{\eta}_{i}}{\tilde{\omega}_{i}}\left(1-e^{-\tilde{\omega}_{i} z}\right) .
$$

The final PDF approximation in (37) has a remarkably simple form as a generalized mixture of $L$ exponentials where $L=$ $n_{R}-N+1$. In section VII, a numerical example is given to show that the performance of a macrodiversity system is very hard to predict without the relevant analytical performance metrics. In the case of (38), we are able to approximate outage probabilities and this is a metric of particular interest in celledge scenarios where outage is of particular concern.

\section{B. Special Cases}

In this section we present the special case where $n_{R}=N$, i.e., the system schedules as many simultaneous users as the number of receive antennas. In this particular scenario, the ZF $\mathrm{CDF}$ analysis in Sec. $\mathrm{V}-\mathrm{A}$ has an intriguing form. From (26), the CF of $\tilde{Z}$ becomes

$$
\phi_{\tilde{Z}}(t) \simeq \frac{\operatorname{Perm}\left(\boldsymbol{Q}_{2}\right)}{|\boldsymbol{D}| \operatorname{Perm}\left(\boldsymbol{D}^{-1} \boldsymbol{Q}_{2}\right)} .
$$

When $n_{R}=N$, the denominator of (39) simplifies to give,

$$
|\boldsymbol{D}| \operatorname{Perm}\left(\boldsymbol{D}^{-1} \boldsymbol{Q}_{2}\right)=\sum_{i=1}^{n_{R}}\left(1-\frac{j t}{\sigma^{2}} P_{i 1}\right) \operatorname{perm}\left(\boldsymbol{Q}_{i 2}\right),
$$

where $\boldsymbol{Q}_{i 2}$ is $\boldsymbol{Q}_{2}$ with the $i^{\text {th }}$ row removed. Then, 39] simplifies to

$$
\begin{aligned}
\phi_{\tilde{Z}}(t) & \simeq \frac{\operatorname{Perm}\left(\boldsymbol{Q}_{2}\right)}{\sum_{i=1}^{n_{R}} \operatorname{perm}\left(\boldsymbol{Q}_{i 2}\right)-\frac{j t}{\sigma^{2}} \sum_{i=1}^{n_{R}} P_{i 1} \operatorname{perm}\left(\boldsymbol{Q}_{i 2}\right)}, \\
& =\frac{\operatorname{Perm}\left(\boldsymbol{Q}_{2}\right)}{\operatorname{Perm}\left(\boldsymbol{Q}_{2}\right)-\frac{j t}{\sigma^{2}} \operatorname{perm}(\boldsymbol{P})} .
\end{aligned}
$$

Inverting the $\mathrm{CF}$ expression in (42) gives the approximate PDF of $\tilde{Z}$ as the simple exponential

$$
\hat{f}_{\tilde{Z}}(z)=\sigma^{2} \theta e^{-\sigma^{2} \theta z},
$$

where $\theta=\operatorname{Perm}\left(\boldsymbol{Q}_{2}\right) / \operatorname{perm}(\boldsymbol{P})$.

\section{High SNR Approximations}

The CF in (24) is a ratio of determinants, where $\boldsymbol{D}=\boldsymbol{I}-$ $\frac{1}{\sigma^{2}} j t \boldsymbol{P}_{1}$. As the SNR grows, $\sigma^{2} \rightarrow 0$ and keeping only the dominant power of $\sigma^{2}$ in (24) gives

$$
\phi_{\tilde{Z}}(t)=\tilde{K}_{0}\left(\frac{\sigma^{2}}{-j t}\right)^{n_{R}-N+1},
$$

where

$$
\tilde{K}_{0}=\frac{1}{\left|\boldsymbol{P}_{1}\right|} E\left\{\frac{\left|\boldsymbol{H}_{2}^{H} \boldsymbol{H}_{2}\right|}{\left|\boldsymbol{H}_{2}^{H} \boldsymbol{P}_{1}^{-1} \boldsymbol{H}_{2}\right|}\right\} .
$$

Following the MGF based approach in [30], the SER of a macrodiversity ZF receiver can be evaluated for $M$-PSK 
modulation as

$$
\tilde{P}_{s}=\frac{1}{\pi} \int_{0}^{T} \mathcal{M}_{\tilde{Z}}\left(-\frac{g}{\sin ^{2} \theta}\right) d \theta .
$$

where $\mathcal{M}_{\tilde{Z}}(s)=\phi_{\tilde{Z}}(-j s), g=\sin ^{2}(\pi / M)$ and $T=$ $\frac{(M-1) \pi}{M}$. Note that linear combinations of equations of the form given in (46) also give SERs for $M$-QAM in the usual way [30]. Substituting (44) in (46) gives

$$
\tilde{P}_{s}^{\infty}=\left(\tilde{G}_{a} \bar{\gamma}^{-\tilde{G}_{d}}+o\left(\bar{\gamma}^{-\tilde{G}_{d}}\right),\right.
$$

where $o($.$) is the standard little-o notation and the average$ SNR is $\bar{\gamma}=\frac{1}{\sigma^{2}}$. The diversity gain and array gain in (47) are given by

$$
\tilde{G}_{d}=n_{R}-N+1, \quad \tilde{G}_{a}=\left(\tilde{K}_{0} \tilde{\mathcal{I}}\right)^{-1 /\left(n_{R}-N+1\right)},
$$

where $\tilde{\mathcal{I}}$ is given by

$$
\tilde{\mathcal{I}}=\frac{1}{\pi} \int_{0}^{T}\left(\frac{\sin ^{2} \theta}{g}\right)^{\left(n_{R}-N+1\right)} d \theta .
$$

The high SNR expression derived in (47) will be exact, if and only if $\tilde{K}_{0}$ is exact. An exact calculation of $\tilde{K}_{0}$ for the $N=2$ case is presented in [26] and shown to have a complex expression. This work suggests that in the general case an exact calculation is likely to be either excessively complicated or intractable. Hence, in this work, we use a Laplace type approximation for $\tilde{K}_{0}$ in (45) to obtain a more compact and insightful expression. Hence, we use the following approximation

$$
\tilde{K}_{0} \simeq \frac{1}{\left|\boldsymbol{P}_{1}\right|} \frac{E\left\{\left|\boldsymbol{H}_{2}^{H} \boldsymbol{H}_{2}\right|\right\}}{E\left\{\left|\boldsymbol{H}_{2}^{H} \boldsymbol{P}_{1}^{-1} \boldsymbol{H}_{2}\right|\right\}} .
$$

Using Lemma 1, 49) is given by

$$
\tilde{K}_{0} \simeq \frac{\operatorname{Perm}\left(\boldsymbol{Q}_{2}\right)}{\left|\boldsymbol{P}_{1}\right| \operatorname{Perm}\left(\boldsymbol{P}_{1}^{-1} \boldsymbol{Q}_{2}\right)} .
$$

Note that when $N=2$, approximate $\tilde{K}_{0}$ has simpler expression [14], which gives

$$
\tilde{K}_{0} \simeq \frac{\operatorname{Tr}\left(\boldsymbol{P}_{2}\right)}{\left|\boldsymbol{P}_{1}\right| \operatorname{Tr}\left(\boldsymbol{P}_{1}^{-1} \boldsymbol{P}_{2}\right)} .
$$

The high SNR SER approximation in (47) has the useful property that all the dependence on $\mathrm{P}$ is encapsulated in the $\tilde{K}_{0}$ metric in (50). Hence, $\tilde{K}_{0}$ acts as a stand-alone performance metric as shown in the numerical example in Sec. VII. This feature has implications for systems where only long-term CSI is available for scheduling. Here, $\tilde{K}_{0}$ can be used as a scheduling metric [32] as it is a one-to-one function of the approximate SER. Such situations include systems with rapidly changing channels, systems where CSI exchange is too expensive and systems with large numbers of sources and/or receivers. In all these cases, long term CSI based scheduling may be preferable due to the overheads, delays and errors implicit in obtaining instantaneous CSI [31].
VI. MMSE ANALYSIS

\section{A. CDF Approximations}

In this section, we derive the approximate $\mathrm{CDF}$ of the output SINR of an MMSE receiver and a high SNR approximation to the SER. Let $Z$ be the output SINR of an MMSE receiver given by (5). Following the same procedure as in the $\mathrm{ZF}$ analysis, the $\mathrm{CF}$ of $\mathrm{Z}$ is

$$
\phi_{Z}(t)=E\left\{e^{j t Z}\right\}=E\left\{e^{j t \boldsymbol{h}_{1}^{H} \boldsymbol{R}^{-1} \boldsymbol{h}_{1}}\right\} .
$$

Next, the CF conditioned on $\boldsymbol{H}_{2}$ becomes [14]

$$
\phi_{Z}\left(t \mid \boldsymbol{H}_{2}\right)=\frac{1}{\left|\boldsymbol{I}-j t \boldsymbol{R}^{-1} \boldsymbol{P}_{1}\right|} .
$$

Since $\boldsymbol{R}=\sigma^{2} \boldsymbol{I}+\boldsymbol{H}_{2} \boldsymbol{H}_{2}^{H}$, the conditional CF in (53) becomes

$$
\begin{aligned}
\phi_{Z}\left(t \mid \boldsymbol{H}_{2}\right) & =\frac{1}{\left|\boldsymbol{I}-j t\left(\sigma^{2} \boldsymbol{I}+\boldsymbol{H}_{2} \boldsymbol{H}_{2}^{H}\right)^{-1} \boldsymbol{P}_{1}\right|} \\
& =\frac{\left|\sigma^{2} \boldsymbol{I}+\boldsymbol{H}_{2} \boldsymbol{H}_{2}^{H}\right|}{\left|\sigma^{2} \boldsymbol{I}-j t \boldsymbol{P}_{1}+\boldsymbol{H}_{2} \boldsymbol{H}_{2}^{H}\right|} .
\end{aligned}
$$

Using the determinant identity, $\left|\boldsymbol{I}+\boldsymbol{X} \boldsymbol{X}^{H}\right|=\left|\boldsymbol{I}+\boldsymbol{X}^{H} \boldsymbol{X}\right|$, where the rank of the identity matrix is obvious from the context, in (55) along with some simple algebra, we get

$$
\phi_{Z}\left(t \mid \boldsymbol{H}_{2}\right)=\frac{\left|\sigma^{2} \boldsymbol{I}+\boldsymbol{H}_{2}^{H} \boldsymbol{H}_{2}\right|}{|\boldsymbol{D}|\left|\sigma^{2} \boldsymbol{I}+\boldsymbol{H}_{2}^{H} \boldsymbol{D}^{-1} \boldsymbol{H}_{2}\right|},
$$

where $\boldsymbol{D}=\boldsymbol{I}-\frac{1}{\sigma^{2}} j t \boldsymbol{P}_{1}$. Note the similarity of (56) with [14, eq. 14]. Then, the full $\mathrm{CF}$ can be solved by averaging the conditional CF in (56) over $\boldsymbol{H}_{2}$. Hence,

$$
\phi_{Z}(t)=\frac{1}{|\boldsymbol{D}|} E\left\{\frac{\left|\sigma^{2} \boldsymbol{I}+\boldsymbol{H}_{2}^{H} \boldsymbol{H}_{2}\right|}{\left|\sigma^{2} \boldsymbol{I}+\boldsymbol{H}_{2}^{H} \boldsymbol{D}^{-1} \boldsymbol{H}_{2}\right|}\right\} .
$$

Using a similar approach as in the $\mathrm{ZF}$ analysis we approximate (57) to get

$$
\phi_{Z}(t) \simeq \frac{1}{|\boldsymbol{D}|} \frac{E\left\{\left|\sigma^{2} \boldsymbol{I}+\boldsymbol{H}_{2}^{H} \boldsymbol{H}_{2}\right|\right\}}{E\left\{\left|\sigma^{2} \boldsymbol{I}+\boldsymbol{H}_{2}^{H} \boldsymbol{D}^{-1} \boldsymbol{H}_{2}\right|\right\}} .
$$

In Appendix B we obtain the expectation in the numerator of (58) as

$$
E\left\{\left|\sigma^{2} \boldsymbol{I}+\boldsymbol{H}_{2}^{H} \boldsymbol{H}_{2}\right|\right\}=\sum_{k=0}^{N-1} \sum_{\sigma} \operatorname{Perm}\left(\left(\boldsymbol{Q}_{2}\right)^{\sigma_{k, N-1}}\right)\left(\sigma^{2}\right)^{N-k-1} .
$$

Appendix $[$ gives the denominator of $(58)$ as

$$
|\boldsymbol{D}| E\left\{\left|\sigma^{2} \boldsymbol{I}+\boldsymbol{H}_{2}^{H} \boldsymbol{D}^{-1} \boldsymbol{H}_{2}\right|\right\}=\sum_{i=0}^{n_{R}}(-j t)^{i} \varphi_{i},
$$


where

$$
\varphi_{i}=\sum_{k=0}^{N-1} \hat{\varphi}_{i k}\left(\sigma^{2}\right)^{N-i-k-1}
$$

and $\hat{\varphi}_{i k}$ is given in (108). Substituting (59) and (60) in (58) we get,

$$
\begin{aligned}
\phi_{Z}(t) & \simeq \frac{\sum_{k=0}^{N-1} \sum_{\sigma} \operatorname{Perm}\left(\left(\boldsymbol{Q}_{2}\right)^{\sigma_{k, N-1}}\right)\left(\sigma^{2}\right)^{N-k-1}}{\sum_{i=0}^{n_{R}}(-j t)^{i} \varphi_{i}} \\
& =\frac{\Theta\left(\boldsymbol{Q}_{2}\right)}{\varphi_{n_{R}} \sum_{i=0}^{n_{R}}\left(\frac{\varphi_{i}}{\varphi_{n_{R}}}\right)(-j t)^{i}} \\
& =\frac{\Theta\left(\boldsymbol{Q}_{2}\right)}{\varphi_{n_{R}} \prod_{i=1}^{n_{R}}\left(\omega_{i}-j t\right)},
\end{aligned}
$$

where $\tilde{\omega}_{i}>0$ for all $i$ is from Descarte's rule of signs and

$$
\Theta\left(\boldsymbol{Q}_{2}\right)=\sum_{k=0}^{N-1} \sum_{\sigma} \operatorname{Perm}\left(\left(\boldsymbol{Q}_{2}\right)^{\sigma_{k, N-1}}\right)\left(\sigma^{2}\right)^{N-k-1} .
$$

The final expression for $\phi_{Z}(t)$ then becomes

$$
\phi_{Z}(t) \simeq \frac{\Theta\left(\boldsymbol{Q}_{2}\right)}{\varphi_{n_{R}}} \sum_{i=1}^{n_{R}} \frac{\eta_{i}}{\omega_{i}-j t}
$$

where

$$
\eta_{i}=\frac{1}{\prod_{k \neq i}^{n_{R}}\left(\omega_{k}-\omega_{i}\right)} .
$$

As in the ZF analysis, the PDF and CDF of $Z$ can be computed using the identity in $[25$, eq. $7,3.382]$. Finally we get the approximate PDF of $Z$ as

$$
\hat{f}_{Z}(z)=\frac{\Theta\left(\boldsymbol{Q}_{2}\right)}{\varphi_{n_{R}}} \sum_{i=1}^{n_{R}} \eta_{i} e^{-\omega_{i} z}
$$

and the CDF of $Z$ becomes

$$
\hat{F}_{Z}(z)=\frac{\Theta\left(\boldsymbol{Q}_{2}\right)}{\varphi_{n_{R}}} \sum_{i=1}^{n_{R}} \frac{\eta_{i}}{\omega_{i}}\left(1-e^{-\omega_{i} z}\right) .
$$

In contrast to (37), where the ZF SNR is a generalized mixture of $L$ exponentials, (68) can be identified as a generalized mixture of $n_{R} \geq L$ exponentials. Since the MMSE SINR has more mixing parameters $\left(n_{R}\right.$ rather than $\left.L\right)$ it might be expected that these increased degrees of freedom will result in a better approximation. Alternatively, the more concise $\mathrm{ZF}$ result, which provides a lower bound on the MMSE performance, can be used to provide a simpler expression for use in system design and understanding.

\section{B. High SNR Approximations}

The CF in (58) is a ratio of determinants. As the SNR grows, $\sigma^{2} \rightarrow 0$ and keeping only the dominant power of $\sigma^{2}$ in 58 gives

$$
\phi(t)=K_{0}(-j t)\left(\frac{\sigma^{2}}{-j t}\right)^{n_{R}-N+1},
$$

where

$$
K_{0}(s)=\frac{1}{\left|\boldsymbol{P}_{1}\right|} E\left\{\frac{\left|\boldsymbol{H}_{2}^{H} \boldsymbol{H}_{2}\right|}{\left|\boldsymbol{H}_{2}^{H} \boldsymbol{P}_{1}^{-1} \boldsymbol{H}_{2}+s \boldsymbol{I}\right|}\right\} .
$$

Hence, from (46), the SER at high SNR becomes

$$
P_{s}^{\infty}=\frac{1}{\pi} \int_{0}^{T}\left(\frac{\sigma^{2} \sin ^{2} \theta}{g}\right)^{n_{R}-N+1} K_{0}\left(\frac{g}{\sin ^{2} \theta}\right) d \theta .
$$

As in the ZF analysis, an exact calculation of $K_{0}$ appears difficult and we use the Laplace-type approximation again to give

$$
K_{0}(s) \simeq \frac{1}{\left|\boldsymbol{P}_{1}\right|} \frac{E\left\{\left|\boldsymbol{H}_{2}^{H} \boldsymbol{H}_{2}\right|\right\}}{E\left\{\left|s \boldsymbol{I}+\boldsymbol{H}_{2}^{H} \boldsymbol{P}_{1}^{-1} \boldsymbol{H}_{2}\right|\right\}} .
$$

From Lemma 1 and (59), we have

$$
\begin{aligned}
K_{0}(s) & =\frac{\operatorname{Perm}\left(\boldsymbol{Q}_{2}\right)}{\left|\boldsymbol{P}_{1}\right|\left(\sum_{i=0}^{N-1} \zeta_{i} s^{N-i-1}\right)}, \\
& =\frac{\operatorname{Perm}\left(\boldsymbol{Q}_{2}\right)\left(\prod_{i=1}^{N-1} \vartheta_{i}\right)}{\left|\boldsymbol{P}_{1}\right|\left(\prod_{i=1}^{N-1} \vartheta_{i}\right) \prod_{i=1}^{N-1}\left(\vartheta_{i}+s\right)}, \\
& =\frac{\operatorname{Perm}\left(\boldsymbol{Q}_{2}\right)}{\left|\boldsymbol{P}_{1}\right| \zeta_{N-1}} \sum_{i=1}^{N-1} \frac{\chi_{i}}{\vartheta_{i}+s},
\end{aligned}
$$

where $\zeta_{i}=\sum_{\sigma} \operatorname{Perm}\left(\left(\boldsymbol{P}_{1}^{-1} \boldsymbol{Q}_{2}\right)^{\sigma_{i, N-1}}\right)$ and $-\vartheta_{i}$ are the roots of $\sum_{i=0}^{N-1} \zeta_{i} s^{N-i-1}$. Since $\zeta_{N-1}=\operatorname{Perm}\left(\boldsymbol{P}_{1}^{-1} \boldsymbol{Q}_{2}\right)$, $K_{0}(s)$ in (76) becomes

$$
K_{0}(s) \simeq \frac{\operatorname{Perm}\left(\boldsymbol{Q}_{2}\right)}{\left|\boldsymbol{P}_{1}\right| \operatorname{Perm}\left(\boldsymbol{P}_{1}^{-1} \boldsymbol{Q}_{2}\right)} \sum_{i=1}^{N-1} \frac{\chi_{i}}{\vartheta_{i}+s},
$$

where

$$
\chi_{i}=\frac{\zeta_{N-1}}{\prod_{k \neq i}^{N-1}\left(\vartheta_{k}-\vartheta_{i}\right)} .
$$

From (72) and (77), we obtain

$$
P_{s}^{\infty}=\left(G_{a} \bar{\gamma}\right)^{-G_{d}}+o\left(\bar{\gamma}^{-G_{d}}\right),
$$

where the diversity order and array gain, $G_{d}$ and $G_{a}$ respectively, are given by

$$
G_{d}=n_{R}-N+1
$$

and

$$
G_{a}=\frac{\operatorname{Perm}\left(\boldsymbol{Q}_{2}\right)}{\left|\boldsymbol{P}_{1}\right| \operatorname{Perm}\left(\boldsymbol{P}_{1}^{-1} \boldsymbol{Q}_{2}\right)} \mathcal{I}(\boldsymbol{P})
$$

where

$$
\mathcal{I}(\boldsymbol{P})=\frac{1}{\pi g^{L}} \sum_{i=1}^{N-1} \frac{\chi_{i}}{\vartheta_{i}} \int_{0}^{T} \frac{\left(\sin ^{2} \theta\right)^{L+1}}{\frac{g}{\vartheta_{i}}+\sin ^{2} \theta} d \theta .
$$


The integrals in (82) can be solved in closed form as in [26]. Hence, the final result becomes

$$
\mathcal{I}(\boldsymbol{P})=\frac{1}{g^{L}} \sum_{i=1}^{N-1} \frac{\chi_{i}}{\vartheta_{i}} J_{L+1}\left(T, \frac{g}{\vartheta_{i}}\right),
$$

where

$$
J_{m}(c, a)=\frac{1}{\pi} \int_{0}^{c} \frac{\sin ^{2 m} \theta}{a+\sin ^{2} \theta} d \theta
$$

\section{Simulations AND Numerical Results}

In this section, we simulate the macrodiversity system shown in Fig. 3, where three base stations (BSs) collaborate via a central backhaul processing (BPU) in the shaded three sector cluster. This simulation environment was also used in [14] and is sometimes referred to as an edge-excited cell. We consider the three BS scenario having either a single antenna or two antennas each to give $n_{R}=3$ or $n_{R}=6$ respectively. In the shaded coverage area of this edge-excited cell, we drop three or four users uniformly in space giving $N=3$ or $N=4$. For each user, lognormal shadow fading and path loss is considered, where the standard deviation of the shadowing is $8 \mathrm{~dB}$ and the path loss exponent is $\gamma=3.5$. The transmit power of the sources is scaled so that the best signal received at the three BS locations is greater than $3 \mathrm{~dB}$ at least $95 \%$ of the time. Even though the analysis in this paper is valid for any set of channel powers, the above methodology allows us to investigate the accuracy of the performance matrices for realistic sets of channel powers.

In Figs. 4 5 and 6, the case of three single antenna users and three distributed BSs with a single receiver antenna is considered. Here, we investigate both the approximate SINR distributions and the approximate SER results for an MMSE receiver. In Fig. 4, the approximate CDFs of the output SINR are plotted alongside the simulated CDFs. Results are shown for four random drops and, the results are for a particular user (the first of the three). The agreement between the CDFs is shown to be excellent. Note that this agreement is good across all drops, from D1 which has a very poor SINR performance to D4 with a much higher SINR performance. The use of physically motivated drops rather than ad-hoc scenarios is useful as it assesses the accuracy of the analysis in plausible channel conditions.

In Fig. 5] the approximate SER curve is plotted alongside the simulated values. Results are shown for three drops and QPSK modulation. The agreement between the SER results is shown to be excellent across all three drops at SERs below $10^{-2}$. Again, this agreement is observed over a wide range with D1 having much higher SERs than D3. In Fig. 5 and also in Figs. 7] 8 the SER is plotted against the transmit SNR, $\bar{\gamma}$. This is chosen instead of the receive SNR to separate the curves so that the drops are visible and are not all superimposed, which tends to happen when SER is plotted against receive SNR.

In Fig. 6, the approximate CDFs of the SNR are plotted alongside the simulated CDFs for a $\mathrm{ZF}$ receiver. Results are shown for four random drops. This is the companion plot to Fig. 4 with the same system but a ZF receiver rather than an MMSE receiver. The accuracy of the results in Fig. 4 and Fig.
6 is interesting, especially when you observe that the Fig. 4 analysis uses (69), a simple mixture of 3 exponentials, and Fig. 6 uses (38) which is a single exponential in this case.

In Fig. 7 and 8 , the case of four single antenna users and six distributed receive antennas (two at each BS location) is considered. High SNR SER curves are plotted alongside the simulated values. Results are shown for both MMSE (Fig. 7) and ZF (Fig. 8) with QPSK modulation. The agreement between the simulated SER and the high SNR approximation is shown to be less accurate than in Fig. 5, with very close agreement requiring low error rates around $10^{-4}$. This is unsurprising, as the greater number of system dimensions gives greater freedom for the channel powers to vary substantially over the links.

The results in Fig. 8 are very informative concerning macrodiversity combining and highlight the difficulties in predicting performance from the $\boldsymbol{P}$ matrix. Consider the simple SIR metric given by the sum of the first column of $\boldsymbol{P}$ (the total long term received power from the desired user 1) divided by the sum of columns 2,3 and 4 (the total long term interfering power). In drops D1, D2 and D 3 the SIR is $-19 \mathrm{~dB},-2.5 \mathrm{~dB}$ and $6.5 \mathrm{~dB}$. As the SIR increases, the SER in Fig. 8 drops. This is also shown by the $\tilde{K}_{0}$ metric in (50) which gives 17000,323 and 13 for drops D1, D2 and D3. As SER increases with $\tilde{K}_{0}$ both the $\tilde{K}_{0}$ metric and the simple SIR metric give the same performance ranking with D3 the best and D1 the worst. The fourth drop, D4, is the interesting case. Here, the SIR is $10 \mathrm{~dB}$, which is lower than both D2 and D3. Hence, from Fig. 8 D4 has a better SER performance than D2 and D3 despite having a worse SIR. In order to understand this, consider the $P$ matrix for drop D4,

$$
\boldsymbol{P}_{D 4}=\left(\begin{array}{cccc}
0.2061 & 1.3941 & 1.1034 & 4.6938 \\
0.2061 & 1.3941 & 1.1034 & 4.6938 \\
2.2923 & 16.8146 & 0.0857 & 0.6790 \\
2.2923 & 16.8146 & 0.0857 & 0.6790 \\
0.8361 & 3.4834 & 2.8181 & 0.6700 \\
0.8361 & 3.4834 & 2.8181 & 0.6700
\end{array}\right)
$$

Again, it is difficult to see why D4 performs the best, since the strongest long term power is on antennas $3 \& 4$ which also have the strongest interference from user 2. Performance is clearly a complex issue and is strongly related to the noise inflation caused by the inverse operation in ZF reception (see (8)). To verify this behavior, Fig. 9 presents SNR curves for user 1 for drops D3 and D4 at $\bar{\gamma}=20 \mathrm{~dB}$. As can be seen, the lower tail for D3 is higher than for D4 and this explains the increased SER. Although a simple exploration of the $\boldsymbol{P}$ matrix makes it difficult to predict D4 as the highest performing drop, the $\tilde{K}_{0}$ metric captures this behavior as $\tilde{K}_{0}=1.3$, the lowest value for all drops. In summary, the performance as a function of $\boldsymbol{P}$ is difficult to predict without the analytical tools provided and here the metric $\tilde{K}_{0}$ is particularly useful.

\section{CONCLUSION}

The performance of MMSE and ZF receivers is well-known in microdiversity systems where the receive antennas are colocated. However, in the macrodiversity case, closed form performance analysis is a long-standing, unsolved research 


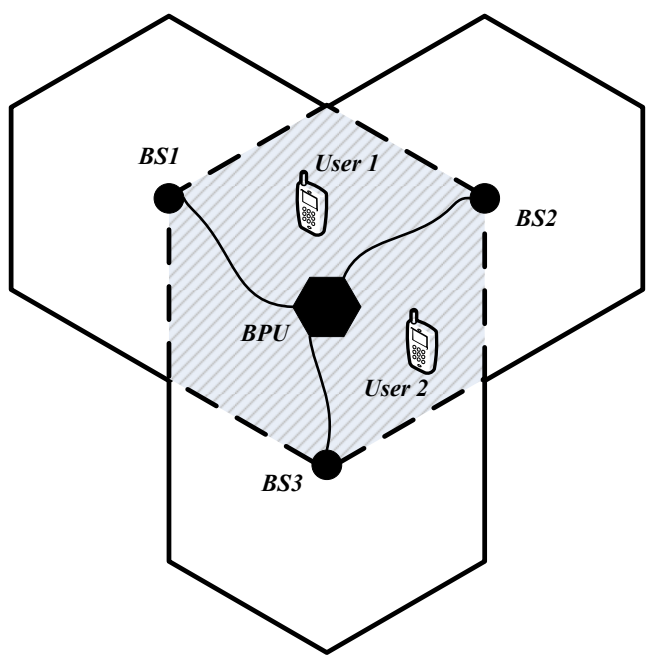

Fig. 3. Network MIMO/edge-excited cell scenario where three base stations serve users in a three-sector cluster. To reduce clutter, only two users are shown.

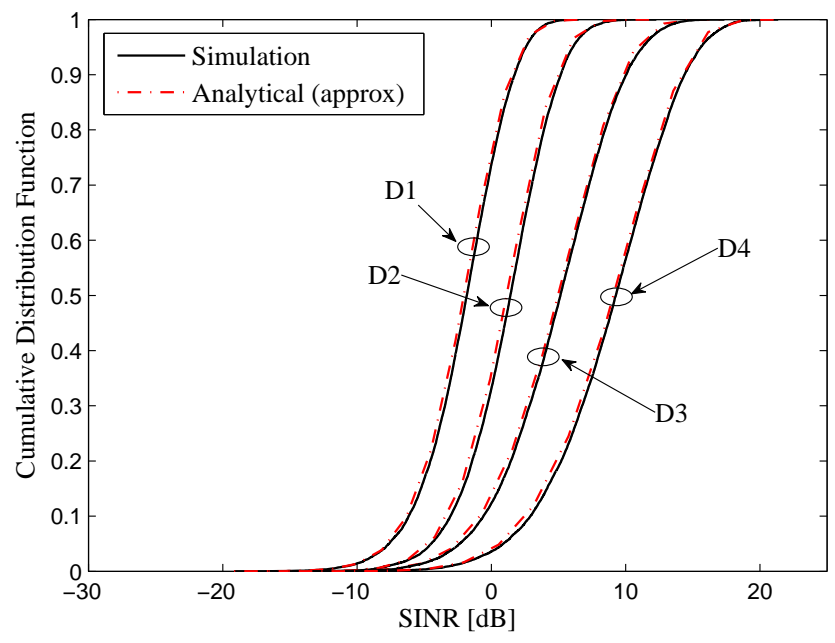

Fig. 4. Approximate and simulated SINR CDF results for the $N=3$, $n_{R}=3$ scenario. Results are shown for the first of three users for four arbitrary drops and a MMSE receiver.

problem. In this paper, we make the progress towards solving this problem for the general case of an arbitrary number of transmit and receive antennas. The analysis is based on a derivation which targets the characteristic function of the output SINR. This leads to an expected value which is highly complex in its exact form, but can be simplified by the use of an extended Laplace type approximation. The SINR distribution is shown to have a remarkably simple form as a generalized mixture of exponentials. Also, the asymptotic SER results produce a remarkably compact metric which captures a large part of the functional relationship between the macrodiversity power profile and SER.

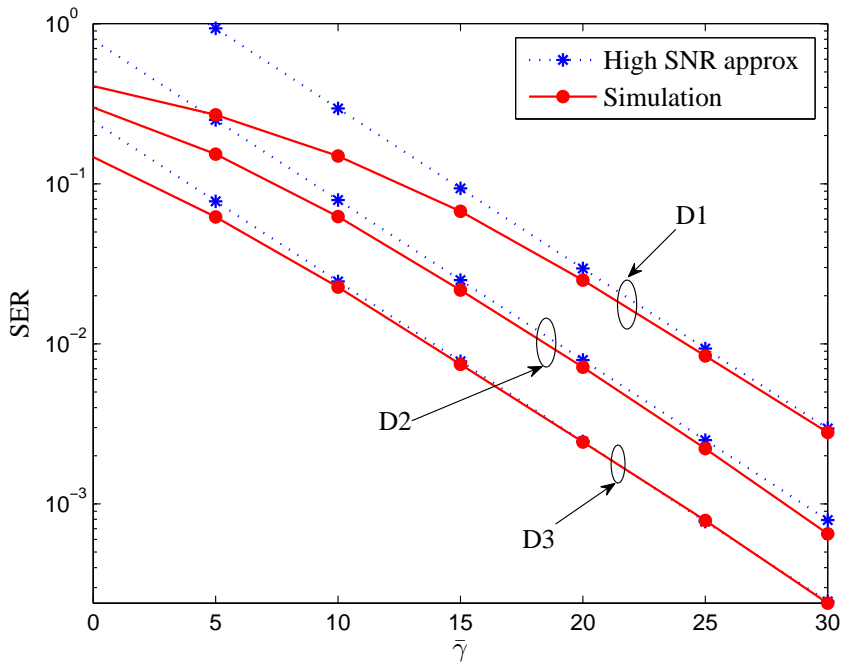

Fig. 5. Approximate and simulated SER results for the $N=3, n_{R}=3$ scenario with QPSK modulation. Results are shown for the first of the three users for three arbitrary drops and a MMSE receiver.

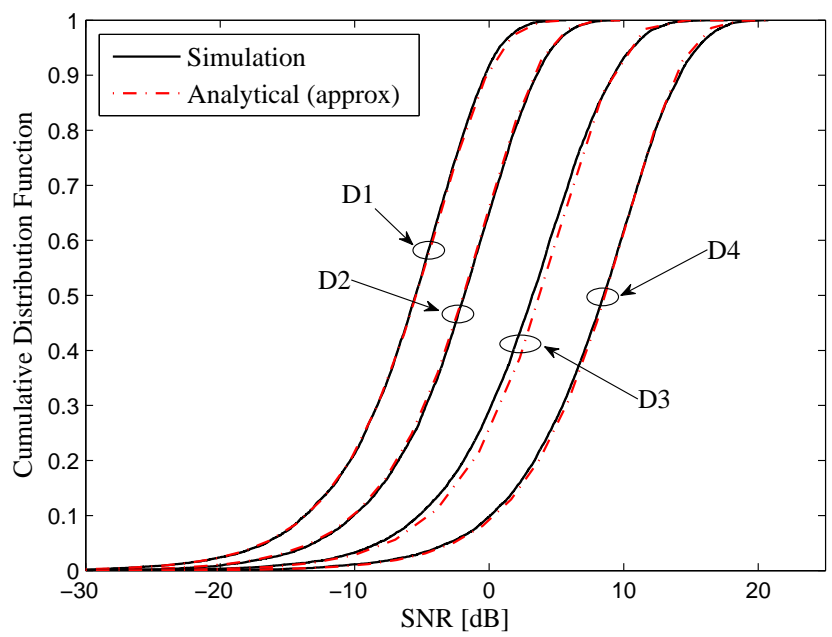

Fig. 6. Approximate and simulated SNR CDF results for the $N=3, n_{R}=3$ scenario. Results are shown for the first of three users for four arbitrary drops and a $\mathrm{ZF}$ receiver.

\section{APPENDIX A \\ Calculation of $|\boldsymbol{D}| \operatorname{Perm}\left(\boldsymbol{D}^{-1} \boldsymbol{Q}_{2}\right)$}

The permanent of the denominator in (26) can be expanded as

$$
\operatorname{Perm}\left(\boldsymbol{D}^{-1} \boldsymbol{Q}_{2}\right)=\sum_{\sigma} \operatorname{perm}\left(\left(\boldsymbol{D}^{-1} \boldsymbol{Q}_{2}\right)_{\sigma_{N-1, n_{R}}}^{\{N-1\}}\right),
$$

where $\sigma_{N-1, n_{R}}$ is an ordered subset of $\left\{n_{R}\right\}=$ $\left\{1,2, \ldots, n_{R}\right\}$ of length $N-1$ and the sum is over all $\left(\begin{array}{c}n_{R} \\ N-1\end{array}\right)$ such subsets. Noting the fact that $\operatorname{perm}(\boldsymbol{\Sigma} \boldsymbol{X})=|\boldsymbol{\Sigma}| \operatorname{perm}(\boldsymbol{X})$, for a square diagonal matrix $\Sigma$ and (14), (86) can be further 


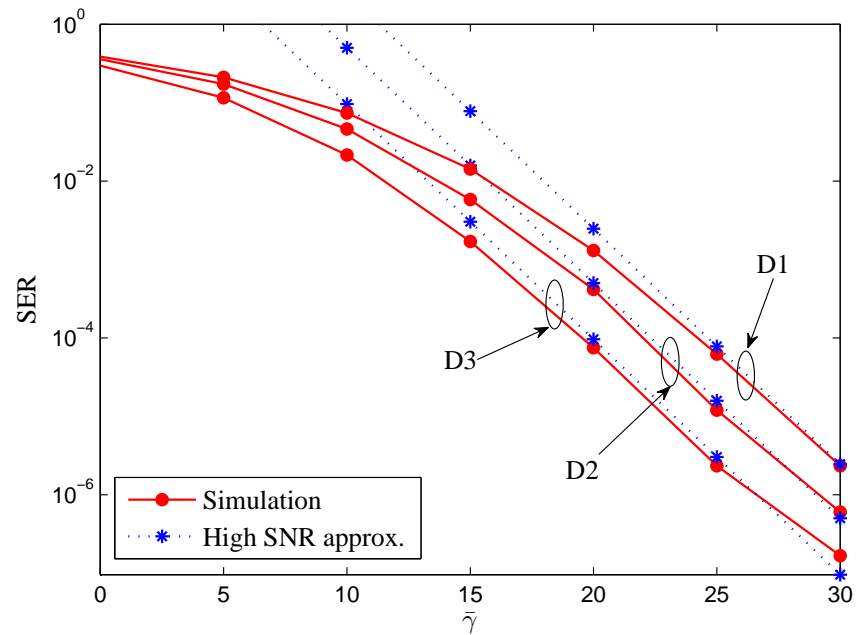

Fig. 7. Approximate and simulated SER results for $N=4, n_{R}=6$, i.e., two receive antennas at each BS with QPSK modulation. Results are shown for the first of four users for three arbitrary drops and a MMSE receiver.

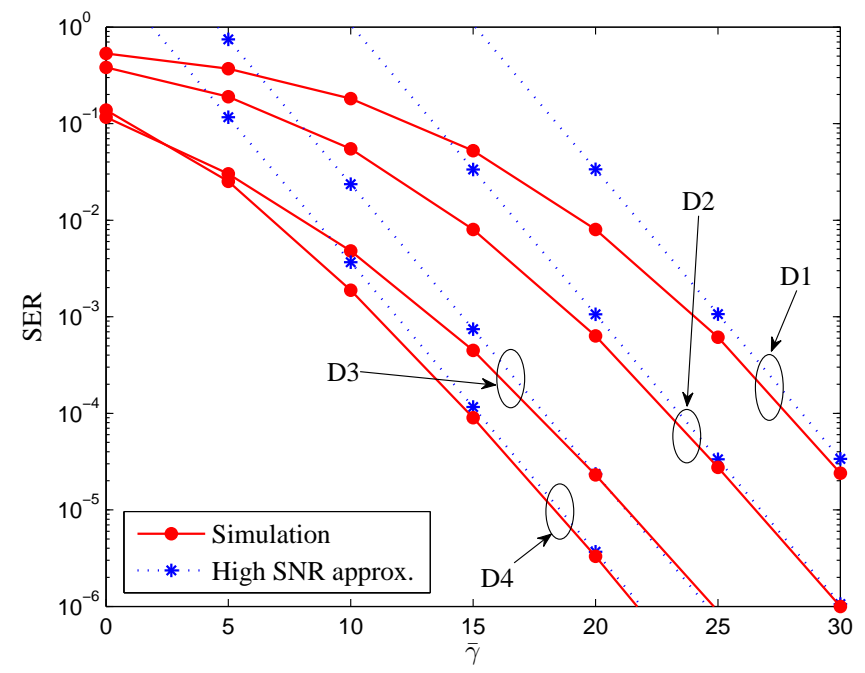

Fig. 8. Approximate and simulated SER results for the $N=4, n_{R}=6$, i.e., two receive antenna at each BS scenario with QPSK modulation. Results are shown for the first of four users for four arbitrary drops and a ZF receiver.

simplified to give

$$
\operatorname{Perm}\left(\boldsymbol{D}^{-1} \boldsymbol{Q}_{2}\right)=\sum_{\sigma} \frac{\operatorname{perm}\left(\left(\boldsymbol{Q}_{2}\right)_{\sigma_{N-1, n_{R}}}^{\{N-1\}}\right)}{\left|\boldsymbol{D}_{\sigma_{N-1, n_{R}}}^{\{N-1\}}\right|}
$$

Using (87), the denominator in (26) becomes

$$
\begin{aligned}
|\boldsymbol{D}| \operatorname{Perm}\left(\boldsymbol{D}^{-1} \boldsymbol{Q}_{2}\right)= & \sum_{\sigma}\left|\boldsymbol{D}_{\bar{\sigma}_{L, n_{R}} \mid}\right| \\
& \times \operatorname{perm}\left(\left(\boldsymbol{Q}_{2}\right)_{\sigma_{N-1, n_{R}}}^{\{N-1\}}\right),
\end{aligned}
$$

where $\bar{\sigma}_{L, n_{R}}$ is the ordered subset of length $L$ of $\left\{1,2, \ldots, n_{R}\right\}$ which does not belong to $\sigma_{N-1, n_{R}}$ and $L=$

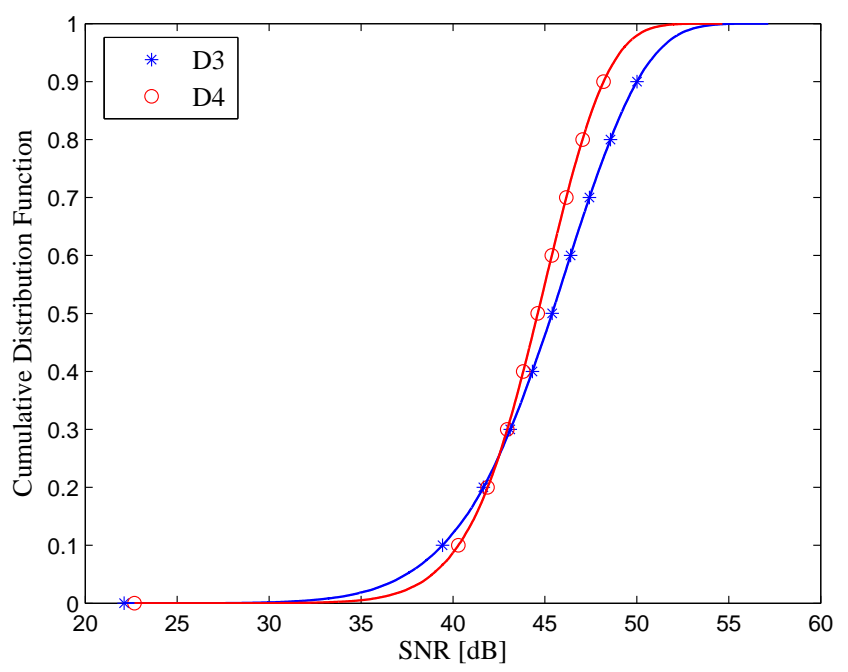

Fig. 9. Simulated SNR CDF results for the $N=4, n_{R}=6$, i.e., two receive antenna at each BS scenario. Results are shown for the first of four users for D3 and D4 drops in Fig. 8 and a ZF receiver.

$n_{R}-N+1$. Expanding $\left|\boldsymbol{D}_{\bar{\sigma}_{L, n_{R}}}\right|$ gives

$$
\left|\boldsymbol{D}_{\bar{\sigma}_{L, n_{R}}}\right|=\sum_{i=0}^{L}\left(\frac{-j t}{\sigma^{2}}\right)^{i} \operatorname{Tr}_{i}\left(\left(\boldsymbol{P}_{1}\right)_{\bar{\sigma}_{L, n_{R}}}\right) .
$$

Substituting (89) in (88) gives the desired result

$$
|\boldsymbol{D}| \operatorname{Perm}\left(\boldsymbol{D}^{-1} \boldsymbol{Q}_{2}\right)=\sum_{i=0}^{L}(-j t)^{i} \tilde{\varphi}_{i},
$$

where

$$
\tilde{\varphi}_{i}=\sum_{\sigma} \operatorname{Tr}_{i}\left(\left(\boldsymbol{P}_{1}\right)_{\bar{\sigma}_{L, n_{R}}}\right) \operatorname{perm}\left(\left(\boldsymbol{Q}_{2}\right)_{\sigma_{N-1, n_{R}}}^{\{N-1\}}\right)\left(\sigma^{2}\right)^{-i} .
$$

$$
\begin{aligned}
& \text { Appendix B } \\
& \text { Calculation OF } E\left\{\left|\sigma^{2} \boldsymbol{I}+\boldsymbol{H}_{2}^{H} \boldsymbol{H}_{2}\right|\right\}
\end{aligned}
$$

Similar expectation results for random determinants can also be found in [16]. However, for completeness we present the particular result needed for the MMSE analysis here. Let $\lambda_{1}, \lambda_{2}, \ldots, \lambda_{N-1}$ be the ordered eigenvalues of $\boldsymbol{H}_{2}^{H} \boldsymbol{H}_{2}$. Since $n_{R} \geq N$, all eigenvalues are non zero. Then

$$
\begin{aligned}
E\left\{\left|\sigma^{2} \boldsymbol{I}+\boldsymbol{H}_{2}^{H} \boldsymbol{H}_{2}\right|\right\} & =E\left\{\prod_{i=1}^{N-1}\left(\sigma^{2}+\lambda_{i}\right)\right\} \\
& =E\left\{\sum_{i=0}^{N-1} \operatorname{Tr}_{i}\left(\boldsymbol{H}_{2}^{H} \boldsymbol{H}_{2}\right)\left(\sigma^{2}\right)^{N-i-1}\right\},
\end{aligned}
$$

where (93) is from [22, 1.2.9] and [22, 1.2.12]. Therefore, the building block of this expectation is $E\left\{\operatorname{Tr}_{i}\left(\boldsymbol{H}_{2}^{H} \boldsymbol{H}_{2}\right)\right\}$. From [22, 1.2.12],

$$
\operatorname{Tr}_{i}\left(\boldsymbol{H}_{2}^{H} \boldsymbol{H}_{2}\right)=\sum_{\sigma}\left|\left(\boldsymbol{H}_{2}^{H} \boldsymbol{H}_{2}\right)_{\sigma_{i, N-1}}\right| .
$$


Therefore, from Lemma 1

$$
E\left\{\operatorname{Tr}_{i}\left(\boldsymbol{H}_{2}^{H} \boldsymbol{H}_{2}\right)\right\}=\sum_{\sigma} \operatorname{Perm}\left(\left(\boldsymbol{Q}_{2}\right)^{\sigma_{i, N-1}}\right),
$$

where the $n_{R} \times(N-1)$ matrix, $\boldsymbol{Q}_{2}$, is given by

$$
E\left\{\boldsymbol{H}_{2} \circ \boldsymbol{H}_{2}\right\}=\boldsymbol{Q}_{2} .
$$

Then, the final expression becomes

$$
E\left\{\left|\sigma^{2} \boldsymbol{I}+\boldsymbol{H}_{2}^{H} \boldsymbol{H}_{2}\right|\right\}=\sum_{i=0}^{N-1} \sum_{\sigma} \operatorname{Perm}\left(\left(\boldsymbol{Q}_{2}\right)^{\sigma_{i, N-1}}\right)\left(\sigma^{2}\right)^{N-i-1} .
$$

$$
\begin{gathered}
\text { Appendix C } \\
\text { Calculation of }|\boldsymbol{D}| E\left\{\left|\sigma^{2} \boldsymbol{I}+\boldsymbol{H}_{2}^{H} \boldsymbol{D}^{-1} \boldsymbol{H}_{2}\right|\right\}
\end{gathered}
$$

As a simple extension of expectation in the numerator of (58), the expectation in the denominator can be calculated using 97 to give

$$
E\left\{\left|\sigma^{2} \boldsymbol{I}+\boldsymbol{H}_{2}^{H} \boldsymbol{D}^{-1} \boldsymbol{H}_{2}\right|\right\}=\sum_{k=0}^{N-1} \psi_{k}(-j t)\left(\sigma^{2}\right)^{N-k-1}
$$

where

$$
\psi_{k}(-j t)=\sum_{\sigma} \operatorname{Perm}\left(\left(\boldsymbol{D}^{-1} \boldsymbol{Q}_{2}\right)^{\sigma_{k, N-1}}\right)
$$

and

$$
\psi_{0}(-j t)=1
$$

The term in (99) can be simplified using (14) and Corollary 1 to obtain

$$
\psi_{k}(-j t)=\sum_{\sigma} \frac{\operatorname{Perm}\left(\left(\boldsymbol{Q}_{2}\right)_{\sigma_{k, n_{R}}}^{\{N-1\}}\right)}{\left|\boldsymbol{D}_{\sigma_{k, n_{R}}}\right|}
$$

Then,

$$
|\boldsymbol{D}| E\left\{\left|\sigma^{2} \boldsymbol{I}+\boldsymbol{H}_{2}^{H} \boldsymbol{D}^{-1} \boldsymbol{H}_{2}\right|\right\}=\sum_{k=0}^{N-1} \xi_{k}(-j t)\left(\sigma^{2}\right)^{N-k-1},
$$

where $\xi_{k}(-j t)=|\boldsymbol{D}| \psi_{k}(-j t)$. From 101), we can get

$$
\xi_{k}(-j t)=\sum_{\sigma}\left|\boldsymbol{D}_{\bar{\sigma}_{n_{R}-k, n_{R}}}\right| \operatorname{Perm}\left(\left(\boldsymbol{Q}_{2}\right)_{\sigma_{k, n_{R}}}^{\{N-1\}}\right),
$$

where $\bar{\sigma}_{n_{R}-k, n_{R}}$ is a length $n_{R}-k$ subset of $\left\{1, \ldots, n_{R}\right\}$ which does not belong to $\sigma_{k, n_{R}}$. Therefore, it is apparent that $\xi_{k}(-j t)$ is a polynomial of degree $n_{R}-k$. It is clear from (102) that, when $\sigma^{2}=0$, 102 collapses to (88). Clearly, $|\boldsymbol{D}| E\left\{\left|\sigma^{2} \boldsymbol{I}+\boldsymbol{H}_{2}^{H} \boldsymbol{D}^{-1} \boldsymbol{H}_{2}\right|\right\}$ is a polynomial of degree $n_{R}$, as $\xi_{0}(-j t)=|\boldsymbol{D}|$ is the highest degree polynomial term in (102). Then,

$$
\left|\boldsymbol{D}_{\bar{\sigma}_{n_{R}-k, n_{R}}}\right|=\sum_{i=0}^{n_{R}-k}\left(\frac{-j t}{\sigma^{2}}\right)^{i} \operatorname{Tr}_{i}\left(\left(\boldsymbol{P}_{1}\right)_{\bar{\sigma}_{n_{R}-k, n_{R}}}\right) .
$$

Hence

$$
\begin{aligned}
\xi_{k}(-j t)=\sum_{\sigma} \sum_{i=0}^{n_{R}-k}\left(\frac{-j t}{\sigma^{2}}\right)^{i} & \operatorname{Tr}_{i}\left(\left(\boldsymbol{P}_{1}\right)_{\bar{\sigma}_{n_{R}-k, n_{R}}}\right) \\
& \times \operatorname{Perm}\left(\left(\boldsymbol{Q}_{2}\right)_{\sigma_{k, n_{R}}}^{\{N-1\}}\right),
\end{aligned}
$$

so that $\xi_{k}(-j t)$ becomes

$$
\begin{aligned}
\xi_{k}(-j t) & =\sum_{i=0}^{n_{R}-k}\left(\frac{-j t}{\sigma^{2}}\right)^{i} \hat{\varphi}_{i k} \\
& =\sum_{i=0}^{n_{R}}\left(\frac{-j t}{\sigma^{2}}\right)^{i} \hat{\varphi}_{i k},
\end{aligned}
$$

where

$$
\hat{\varphi}_{i k}=\sum_{\sigma} \operatorname{Tr}_{i}\left(\left(\boldsymbol{P}_{1}\right)_{\bar{\sigma}_{n_{R}-k, n_{R}}}\right) \operatorname{Perm}\left(\left(\boldsymbol{Q}_{2}\right)_{\sigma_{k, n_{R}}}^{\{N-1\}}\right) \text {, }
$$

and $\hat{\varphi}_{i 0}$ simplifies to give

$$
\hat{\varphi}_{i 0}=\operatorname{Tr}_{i}\left(P_{1}\right) \text {. }
$$

Equation (107) follows from the fact that

$$
\operatorname{Tr}_{i}\left(\left(\boldsymbol{P}_{1}\right)_{\bar{\sigma}_{n_{R}-k, R}}\right)=0 \text { for } i>n_{R}-k .
$$

Therefore, (102) can be written as

$$
\begin{aligned}
|\boldsymbol{D}| E\left\{\left|\sigma^{2} \boldsymbol{I}+\boldsymbol{H}_{2}^{H} \boldsymbol{D}^{-1} \boldsymbol{H}_{2}\right|\right\}=\sum_{k=0}^{N-1} & \sum_{i=0}^{n_{R}}(-j t)^{i} \hat{\varphi}_{i k} \\
& \times\left(\sigma^{2}\right)^{N-i-k-1},
\end{aligned}
$$

which is in turn can be given as

$$
|\boldsymbol{D}| E\left\{\left|\sigma^{2} \boldsymbol{I}+\boldsymbol{H}_{2}^{H} \boldsymbol{D}^{-1} \boldsymbol{H}_{2}\right|\right\}=\sum_{i=0}^{n_{R}}(-j t)^{i} \varphi_{i},
$$

where

$$
\varphi_{i}=\sum_{k=0}^{N-1} \hat{\varphi}_{i k}\left(\sigma^{2}\right)^{N-i-k-1}
$$

\section{REFERENCES}

[1] S. Verdu, Multiuser Detection, 1st ed, Cambridge:Cambridge University Press, 1998.

[2] J. H. Winters, "Optimum combining in digital mobile radio with cochannel interference," IEEE J. Select Areas in Commun., vol.SAC-2, pp. 528-539, July. 1984.

[3] H. Gao, P. J. Smith and M. V. Clark, "Theoretical reliability of MMSE linear diversity combining in Rayleigh-fading additive interference channels," IEEE Trans. Commun., vol. 46, no. 5, pp. 666-672, May. 1998.

[4] J. H. Winters, J. Salz and R. D. Gitlin, "The impact of antenna diversity on the capacity of wireless communication systems," IEEE Trans. Commun., vol. 42, no.2/3/4, pp.1740-1751, Feb/Mar/Apr. 1994.

[5] A. Shah, A. M. Haimovich, M. K. Simon, and M.-S. Alouini, "Exact biterror probability for optimum combining with a Rayleigh fading Gaussian cochannel interferer," IEEE Trans. Commun., vol. 48, pp. 908-912, Jun. 2000.

[6] M. Chiani, M. Z. Win, A. Zanella, R. K. Mallik, and J. H. Winters, "Bounds and approximations for optimum combining of signals in the presence of multiple cochannel interferers and thermal noise," IEEE Trans. Commun., vol. 51, pp. 296-307, Feb. 2003.

[7] D. A. Gore, R. W. Heath, Jr. and A. J. Paulraj, "Transmit selection in spatial multiplexing systems," IEEE Trans. Commun., vol. 6, no.11, pp. 491-493, Nov. 2002 
[8] M. K. Karakayali, G. J. Foschini, and R. A. Valenzuela, "Network coordination for spectrally efficient communications in cellular systems," IEEE Wireless Commun. Mag., vol. 13, no. 4, pp. 56-61, Aug. 2006.

[9] S. Venkatesan, A. Lozano and R. Valenzuela, "Network MIMO: Overcoming intercell interference in indoor wireless systems," IEEE ACSSC, Pacific Grove, California, pp. 83-87, Jul. 2007.

[10] A. Sanderovich, O. Somekh, H.V. Poor and S. Shamai "Uplink macro diversity of limited backhaul cellular network," IEEE Trans. Inform. Theory, vol 55, no. 8, pp. 3457-3478, Aug. 2009.

[11] E. Biglieri, R. Calderbank, A. Constantinides, A. Goldsmith, A. Paulraj and H. V. Poor, MIMO Wireless Communication, 1st ed, New York: Cambridge University Press, 2007.

[12] M. Sawahashi, Y. Kishiyama, A. Morimoto, D. Nishikawa and M Tanno, "Coordinated multipoint transmission/reception techniques for LTE-Advanced,’ IEEE Wireless Commun. Mag., vol. 17, no. 3, pp. 26-34, Aug. 2010.

[13] L. Xiao, L. Dai, H. Zhuang, S. Zhou, Y. Yao, "Information-theoretic capacity analysis in MIMO distributed antenna systems," IEEE VTC, Orlando, Florida, pp. 779-782, 2003.

[14] D. A. Basnayaka, P. J. Smith and P. A. Martin, "Exact dual-user macrodiversity performance with linear receivers in flat Rayleigh fading," IEEE ICC, Ottawa, Canada, pp. 5626-5631, Jun. 2012.

[15] D. A. Basnayaka, P. J. Smith and P. A. Martin, "Symbol error rate performance for macrodiversity maximal ratio combining in flat Rayleigh fading," IEEE AusCTW, Wellington, New Zealand, pp. 25-30, 2012.

[16] D. A. Basnayaka, P. J. Smith and P. A. Martin, "Ergodic Sum Capacity of Macrodiversity MIMO Systems in flat Rayleigh Fading," submitted to IEEE Trans. Inform. Theory.

[17] W. Hachem, P. Loubaton, and J. Najim, "Deterministic equivalents for certain functionals of large random matrices," Ann. App. Probab., vol. 17, no. 3, pp. 875-930, 2007.

[18] S. Chatzinotas, M. Imran, and R. Hoshyar, "On the multicell processing capacity of the cellular MIMO uplink channel in correlated Rayleigh fading environment," IEEE Trans. Wireless Commun., vol. 8, pp. $3704-$ 3715,2008 .

[19] C. K. Wen, K. K. Wong, and J. C. Chen, "Spatially correlated MIMO multiple-access systems with macrodiversity: asymptotic analysis via statistical physics," IEEE Trans. Commun., vol. 55, pp. 477-488, Mar. 2007.

[20] M. V. Clark, L. J. Greenstein, W. K. Kennedy and M. Shafi, "Optimum linear diversity receivers for mobile communication," IEEE Trans. Veh. Technol., vol. 43, no. 1, pp. 47-56, Feb.1994.

[21] H. Minc, Permanants, 1st ed, Massachusetts: Addison-Wesley Publishing Company Inc, 1978.

[22] R. A. Horn and C. R. Johnson, Matrix Analysis. New York: Cambridge Univ. Press, 1985.

[23] H. Cramer, Mathamatical Methods of Statistics, 1st ed, Princeton: Princeton University Press, 1945.

[24] J. G. Proakis, Digital Communications, 4th ed, New York: McGraw-Hill, 2001.

[25] I. S. Gradshteyn and I. M. Ryzhik, Table of Integrals, Series, and Products, 7th ed, Boston: Academic Press, 2000.

[26] D. A. Basnayaka, P. J. Smith and P. A. Martin, "Performance analysis of dual-user macrodiversity MIMO systems with linear receivers in flat Rayleigh fading," submitted to IEEE Trans. Wireless Commun., 2012.

[27] O. Lieberman, "A Laplace approximation to the moments of a ratio of quadratic forms," Biometrika, vol. 81, no. 4, pp. 681-690, Dec 1994.

[28] D. A. Basnayaka, "Macrodiversity MIMO Transceivers," PhD Thesis, Dept. of Electrical and Computer Engineering, University of Canterbury, Jun. 2012.

[29] A. Stuart and J. K. Ord, "Kendall's Advanced Theory of Statistics: Volume 1 Distribution Theory," 6th ed., London, U.K.: Edward Arnold, 1994.

[30] M. K. Simon and M. S. Alouini, Digital Communications over Fading Channels: A Unified Approach to Performance Analysis. New York, NY, USA: Wiley, 2000.

[31] B. Bandemer and S. Visuri, "Capacity-based uplink scheduling using long-term channel knowledge," IEEE ICC, Glasgow, Scotland, pp. 785790, 2007.

[32] P. J. Smith and D. A. Basnayaka, "Multiuser scheduling for radio resource allocation," US provisional patent application 61/648505, filed May 17, 2012

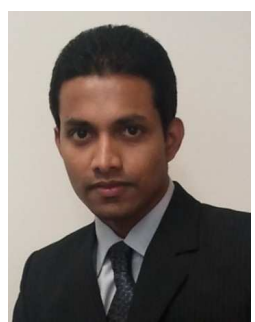

Dushyantha Basnayaka (S'11-M'12) was born in 1982 in Colombo, Sri Lanka. He received the B.Sc.Eng degree with $1^{\text {st }}$ class honors from the University of Peradeniya, Sri Lanka, in Jan 2006. $\mathrm{He}$ is currently working towards for his $\mathrm{PhD}$ degree in Electrical and Computer Engineering at the University of Canterbury, Christchurch, New Zealand. He was an instructor in the Department of Electrical and Electronics Engineering at the University of Peradeniya from Jan 2006 to May 2006. He was a system engineer at MillenniumIT (a member company of London Stock Exchange group) from May 2006 to Jun. 2009. Since Jul. 2009 he is with the communication research group at the University of Canterbury, New Zealand.

D. A. Basnayaka is a recipient of University of Canterbury International Doctoral Scholarship for his doctoral studies at UC. His current research interest includes all the areas of digital communication, especially macrodiversity wireless systems. He holds one pending US patent as a result of his doctoral studies at UC.

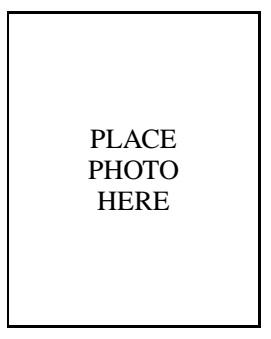

Peter Smith (M'93-SM'01) received the B.Sc degree in Mathematics and the Ph.D degree in Statistics from the University of London, London, U.K., in 1983 and 1988, respectively. From 1983 to 1986 he was with the Telecommunications Laboratories at GEC Hirst Research Centre. From 1988 to 2001 he was a lecturer in statistics at Victoria University, Wellington, New Zealand. Since 2001 he has been a Senior Lecturer and Associate Professor in Electrical and Computer Engineering at the University of Canterbury in New Zealand. Currently, he is a full Professor at the same department.

His research interests include the statistical aspects of design, modeling and analysis for communication systems, especially antenna arrays, MIMO, cognitive radio and relays.

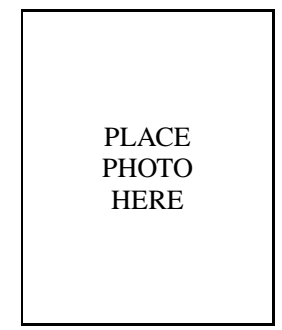

Philippa Martin (S95-M01-SM06) received the B.E. (Hons. 1) and Ph.D. degrees in electrical and electronic engineering from the University of Canterbury, Christchurch, New Zealand, in 1997 and 2001, respectively. From 2001 to 2004, she was a postdoctoral fellow, funded in part by the New Zealand Foundation for Research, Science and Technology (FRST), in the Department of Electrical and Computer Engineering at the University of Canterbury. In 2002, she spent 5 months as a visiting researcher in the Department of Electrical Engineering at the University of Hawaii at Manoa, Honolulu, Hawaii, USA. Since 2004 she has been working at the University of Canterbury as a lecturer and then as a senior lecturer. Currently, she is an Associate Professor at the same department. In 2007, she was awarded the University of Canterbury, College of Engineering young researcher award. She served as an Editor for the IEEE Transactions on Wireless Communications 2005-2008 and regularly serves on technical program committees for IEEE conferences.

Her current research interests include multilevel coding, error correction coding, iterative decoding and equalization, space-time coding and detection, cognitive radio and cooperative communications in particular for wireless communications 